\title{
Neutrino evolution accounting for the longitudinal and transversal matter currents
}

\author{
Alexander Studenikin* \\ Department of Theoretical Physics, Moscow State University, 119992 Moscow, Russia; Joint \\ Institute for Nuclear Research, Dubna 141980, Moscow Region, Russia \\ E-mail: studenikdsrd.sinp.msu.ru
}

\section{Pavel Pustoshny}

Department of Theoretical Physics, Moscow State University, 119992 Moscow, Russia

E-mail: kfreppegmail.com

\begin{abstract}
It is well known that massive neutrinos have nontrivial electromagnetic properties and at least magnetic moments of neutrinos should be nonzero. For many years, until 2004, it was believed that neutrino helicity precession and the corresponding spin oscillations can be induced by the neutrino magnetic moment interactions with the transversal magnetic field. A new and very interesting possibility for neutrino spin (and spin-flavour) oscillations engendered by the neutrino interaction with matter background was proposed and investigated in [四] (also mentioned in [D]). The origin of the new type neutrino spin oscillations $v^{-} \Leftrightarrow v^{+}$in the background matter is the neutrino weak interactions with the transversal matter current $j_{\perp}$ (determined by the contribution $G_{F} j_{\perp}$ to the neutrino evolution Hamiltonian) that generates the corresponding mixing between neutrino states $v_{e}^{-}$and $v_{e}^{+}$. The existence of this effect has been confirmed recently by other authors (see, for instance, [B]]). For historical notes reviewing studies and derivation of the discussed effect see also [四]. In these notes below we give a short flash on the quantum treatment of the effect that, as an example, result with the exact probability of the spin oscillations between the electron neutrino states $v_{e}^{-}$and $v_{e}^{+}$in the transversal matter current $j_{\perp}$.
\end{abstract}

The 39th International Conference on High Energy Physics (ICHEP2018)

4-11 July, 2018

Seoul, Korea

${ }^{*}$ Speaker. 


\section{Neutrino spin oscillations in mass and flavor bases}

Consider [1], 6] two flavour neutrinos with two possible helicities $v_{f}=\left(v_{e}^{+}, v_{e}^{-}, v_{\mu}^{+}, v_{\mu}^{-}\right)^{T}$ in moving media composed of neutrons. The interaction Hamiltonian reads $H_{\text {int }}=f^{\mu} \sum_{l}\left(\bar{v} \gamma_{\mu} \frac{1+\gamma^{5}}{2} v\right)$, where $l=e, \mu, f^{\mu}=G_{F} j^{\mu} / \sqrt{2}, j^{\mu}=n_{n}(1, v) / \sqrt{1-v^{2}}$ is the matter current and $G_{F}$ is Fermi constant of weak interaction. For typical term $\Delta_{\alpha \alpha^{\prime}}^{s \prime^{\prime}}=\left\langle v_{\alpha}^{s}\left|H_{\text {int }}\right| v_{\alpha^{\prime}}^{s^{\prime}}\right\rangle$, that by fixing proper values of $\alpha, s, \alpha^{\prime}$ and $s^{\prime}$ can reproduce all of the elements of the neutrino evolution Hamiltonian $\Delta H^{\text {eff }}$ that accounts for the effect of matter motion, we obtain

$$
\Delta_{\alpha, \alpha^{\prime}}^{s, s^{\prime}}=n \tilde{G} u_{\alpha}^{s T}\left\{\left(1-v_{\|}\right)\left(\begin{array}{ll}
0 & 0 \\
0 & 2
\end{array}\right)+v_{\perp}\left(\begin{array}{cc}
0 & \gamma_{\alpha \alpha}^{-1} \\
\gamma_{\alpha^{\prime} \alpha^{\prime}}^{-1} & 0
\end{array}\right)\right\} u_{\alpha^{\prime}}^{s^{\prime}} \delta_{\alpha, \alpha^{\prime}}
$$

Here, as an example, we consider a particular case of two electron neutrino states $\left(v_{e}^{+}\right.$and $\left.v_{e}^{-}\right)$. The evolution of this system is given by the equation

$$
i \frac{d}{d t}\left(\begin{array}{l}
v_{e}^{+} \\
v_{e}^{-}
\end{array}\right)=\left(\begin{array}{cc}
-\frac{\Delta m^{2}}{4 E} \cos 2 \theta & n \tilde{G} v_{\perp}\left(\frac{\eta}{\gamma}\right)_{e e} \\
n \tilde{G} v_{\perp}\left(\frac{\eta}{\gamma}\right)_{e e} & -\frac{\Delta m^{2}}{4 E} \cos 2 \theta+2 n \tilde{G}\left(1-v_{\|}\right)
\end{array}\right)\left(\begin{array}{l}
v_{e}^{+} \\
v_{e}^{-}
\end{array}\right),\left(\frac{\eta}{\gamma}\right)_{e e}=\frac{\cos ^{2} \theta}{\gamma_{11}}+\frac{\sin ^{2} \theta}{\gamma_{22}}
$$

where $\tilde{G}=\frac{G_{F}}{\sqrt{2}}$ and we have to consider an obvious condition $\Delta m^{2}=0$. In the adiabatic case for the probability of the neutrino spin oscillation $v_{e}^{-} \Leftrightarrow v_{e}^{+}$we get

$$
P_{v_{e}^{+} \rightarrow v_{e}^{-}}=\frac{v_{\perp}^{2}\left(\frac{\eta}{\gamma}\right)_{e e}^{2}}{\left(1-v_{\|}\right)^{2}+v_{\perp}^{2}\left(\frac{\eta}{\gamma}\right)_{e e}^{2}} \sin ^{2}\left(n \tilde{G} \sqrt{\left(1-v_{\|}\right)^{2}+v_{\perp}^{2}\left(\frac{\eta}{\gamma}\right)_{e e}^{2}} x\right) .
$$

It follows that the transversal matter current $j_{\perp}$ generates mixing between two neutrino states whereas the the effect of the longitudinal matter current $j_{\|}$can produce the resonance amplification of the corresponding oscillations.

\section{Acknowledgments}

This work is supported by the Russian Basic Research Foundation grants No. 16-02-01023 and 17-52-53-133.

\section{References}

[1] A. Studenikin, Neutrinos in electromagnetic fields and moving media, Phys. Atom. Nucl. 67 (2004) 993.

[2] A. Studenikin, Neutrino in magnetic fields: From the first studies to the new effects in neutrino oscillations, Frascati Physics Series 34 (2004) 155, hep-ph/0407010.

[3] A. Kartavtsev, G. Raffelt, H. Vogel, Neutrino propagation in media: Flavor-, helicity-, and pair correlations, Phys. Rev. D 91 (2015) 125020.

[4] A. Studenikin, Neutrino spin and spin-flavour oscillations in transversally moving or polarized matter, J. Phys. Conf. Ser. 888 (2017) 012221, arXiv:1610.06563 [hep-ph].

[5] A. Studenikin, From neutrino electromagnetic interactions to spin oscillations in transversal matter currents," PoS NOW 2016 (2017) 070, arXiv:1706.01100 [hep-ph].

[6] P. Pustoshny and A. Studenikin, Neutrino spin and spin-flavour oscillations in transversal matter currents with standard and non-standard interactions, arXiv:1808.00302 [hep-ph]. 